\title{
Kenaf Fibre Reinforced Polypropylene Composites: Effect of Cyclic Immersion on Tensile Properties
}

\author{
W. H. Haniffah, ${ }^{1}$ S. M. Sapuan, ${ }^{1}$ K. Abdan, ${ }^{1}$ M. Khalid, $^{2}$ M. Hasan, ${ }^{3}$ and M. Enamul Hoque ${ }^{4}$ \\ ${ }^{1}$ Department of Mechanical and Manufacturing Engineering, Universiti Putra Malaysia, 43400 Serdang, Malaysia \\ ${ }^{2}$ Division of Manufacturing and Industrial Processes, Faculty of Engineering, University of Nottingham Malaysia Campus, \\ 43500 Semenyih, Malaysia \\ ${ }^{3}$ Department of Materials and Metallurgical Engineering, Bangladesh University of Engineering and Technology, \\ Dhaka 1000, Bangladesh \\ ${ }^{4}$ Department of Biomedical Engineering, King Faisal University, Al-Hofuf, Al-Ahsa 31982, Saudi Arabia
}

Correspondence should be addressed to M. Enamul Hoque; enamul1973@gmail.com

Received 6 January 2015; Revised 24 June 2015; Accepted 5 July 2015

Academic Editor: Antje Potthast

Copyright @ 2015 W. H. Haniffah et al. This is an open access article distributed under the Creative Commons Attribution License, which permits unrestricted use, distribution, and reproduction in any medium, provided the original work is properly cited.

\begin{abstract}
This research studied the degradation of tensile properties of kenaf fibre reinforced polypropylene composites due to cyclic immersion into two different solutions, as well as comparison of the developed composites' tensile properties under continuous and cyclic immersion. Composites with $40 \%$ and $60 \%$ fibre loadings were immersed in tap water and bleach for 4 cycles. Each cycle consisted of 3 days of immersion and 4 days of conditioning in room temperature $\left(28^{\circ} \mathrm{C}\right.$ and $55 \%$ humidity $)$. The tensile strength and modulus of composites were affected by fibre composition, type of liquid of immersion, and number of cycles. The number of immersion cycles and conditioning caused degradation to tensile strength and modulus of kenaf fibre reinforced polypropylene composites. Continuous and cyclic immersion in bleach caused tensile strength of the composites to differ significantly whereas, for tensile modulus, the difference was insignificant in any immersion and fibre loadings. However, continuous immersion in the bleach reduced the tensile strength of composites more compared to cyclic immersion. These preliminary results suggest further evaluation of the suitability of kenaf fibre reinforced polypropylene composites for potential bathroom application where the composites will be exposed to water/liquid in cyclic manner due to discontinuous usage of bathroom.
\end{abstract}

\section{Introduction}

Polymeric composites with various natural reinforcements (e.g., natural fibres, woods, and hybrid fibres) received significant attention in numerous applications including automotive and construction industries [1, 2]. However, the application of natural fibre composites usually intended for interior application; one of the main reasons is degradation of mechanical properties of natural fibre composites in the presence of water. Recent studies [3-8] have reported that the effect of water uptake in natural fiber composites limits their outdoor applications. In general, there are three ways to understand the term "water absorption" such as water diffusing (1) directly into the matrix, (2) through interphase matrix/reinforcements, and (3) by imperfections, like pores and cracks. Once it enters the polymers, it can exist in several ways: as bound water, characterized by strong interaction of the molecule with matrix and free water, present in capillaries and micro cavities within the polymer [9]. When moisture diffuses into composites, it degrades fiber-matrix interfacial bonding, lowers the glass transition temperature, swells, plasticizes, hydrolyzes, and sometimes microcracks the matrix [10]. Although water absorption can cause degradation of mechanical properties, redrying process can help the materials in regaining certain percentages of their mechanical properties [11, 12]. Generally, the moisture diffusion in composite depends on factors such as fiber weight fraction, void volume, viscosity of matrix, chemical treatment of natural fiber, humidity, and temperature [13].

Haniffah et al. reported that, under cyclic immersion, longer duration of immersion and shorter drying exhibit the greatest amount of water retained in kenaf/PP 
composites [14]. The tensile properties were also affected by increasing temperature of water immersion. Generally, it is reported that the tensile properties decreased with increasing immersion time [15-18]. Moreover, the mechanical degradation increased as the number of cyclic immersions increased. However, lesser degree of degradation occurred in composites with fibres modified using coupling agents $[19,20]$. Similar studies on natural fiber reinforced polymeric composites have shown that the sensitivity of certain mechanical and thermal properties to moisture uptake can be significantly reduced by using coupling agents and fiber surface treatments $[21,22]$.

Besides soap, bleach is one of the common chemicals used in bathroom causing physical (e.g., colour) and mechanical changes to the composites that affect the perception of durability of the composites. Kenaf/PP composites experienced degradation by the chemical reagent, bleach [23]. The degradation of kenaf PP composites in bleach solution mostly occurs on kenaf fibres because PP was resistant to bleach attack [24]. The aim of this study is to compare the effect of bleach and water under cyclic immersion on tensile properties of kenaf/polypropylene (PP) composites for potential bathroom application.

\section{Materials and Methods}

2.1. Materials. Kenaf plant (variety V36) used in this study was supplied by National Kenaf and Tobacco Board's (NKTB), Malaysia. Polypropylene, grade TitanPro 6331, was purchased from Titan Chemicals Corp. Sdn., Bhd Johor, Malaysia. Liquids for immersion used in this treatment were tap water ( $\mathrm{pH}$ 7.67) and domestic bleach (Clorox Regular, Malaysia) with active ingredient, for example, sodium hypochlorite $5.25 \%$. Prior to treatment, bleach solution $(\mathrm{pH}$ 11.64) was prepared with $200 \mathrm{~mL}$ of bleach diluted in $1 \mathrm{~L}$ of tap water $(16.17 \% \mathrm{v} / \mathrm{v})$.

2.2. Measurement of Liquid Content. Liquid content in the composite was measured using the following:

$$
\begin{aligned}
& \text { Liquid content }(\%) \\
& \qquad=\left(\frac{\text { Weight }(\mathrm{g})-\text { Initial Weight }(\mathrm{g})}{\text { Initial Weight }(\mathrm{g})}\right) \times 100 \% .
\end{aligned}
$$

Negative value might occur if composites are immersed in bleach especially for longer cycle. This indicates that the bleach causes some parts of composites to dissolve and to be lost in the liquid during conditioning.

2.3. Composite Preparation. The processing of kenaf/PP composites was done at Institute of Tropical Forestry and Forest Product (INTROP), Universiti Putra Malaysia (UPM). The composites were prepared with $40 \%$ and $60 \%$ fibre loadings using corotating extruder (Micromac, Micromagna, Perak, Malaysia). Then, the produced pellets were injected into tensile specimen mould using JSW 85-ton injection moulding machine model J85EM $110 \mathrm{H}$ using injection pressure of $78.72 \mathrm{MPa}$ and injection speed of $48 \mathrm{~mm} / \mathrm{s}$. The temperatures of injection moulding barrel were 195 and $210^{\circ} \mathrm{C}$ for 40 and $60 \%$ fibre loading, respectively. The specimen was prepared following type I of ASTM D638 standard and four replicas were tested for each parameter of treatment.

2.4. Methods of Treatment. All samples underwent 4 cycles of immersion and conditioning with each cycle consisting of 3 days of immersion and 4 days of conditioning. After the fourth day of conditioning, the specimens were tested using universal testing machine (Instron). The other set of specimens underwent immersion for 12 days and was conditioned for 4 days in order to evaluate the tensile properties of the composites under cyclic and continuous immersion. Three determined factors in this study were composition ( $40 \%$ and $60 \%$ fibre loadings), liquid of immersion (water and bleach), and cycle of immersion (the 0 th, the 1st, the $2 \mathrm{nd}$, the $3 \mathrm{rd}$, and the 4 th cycle). Two statistical analysis methods, ANOVA FTest and Fisher's least significant difference (LSD), were used to determine the significant difference between experimental results.

\section{Results and Discussion}

3.1. Liquid Content in Composite. Liquid content in composite was the general term used in this study to describe the amount of liquid in composite at certain time regardless of whether composite was in immersion or conditioning stage. Liquid absorption was the amount of liquid absorbed by composite during immersion stage, while liquid retention was amount of liquid retained by composites during conditioning stage. The results of sorption pattern are presented in Figure 1 with all factors included in the graph. Composites were immersed in liquid and conditioned at room temperature for 4 cycles. During the first cycle of immersion, two distinguishable patterns were observed for composites with 40 and $60 \%$ fibre loading. Liquid of immersion (bleach and water) caused the patterns of liquid content to differentiate from each other with increase in cycle. The difference in pattern of liquid content was more apparent at the end of last cycle in composites with $60 \%$ fibre loading than composites with $40 \%$ fibre loading. It is clear that, even after the conditioning, some of the water absorbed by the fibers could not be removed from the composites and the amount of liquid retained inside the composite also increased with increase in immersion cycle. On the other hand, the amount of liquid retained by the composite during bleach immersion was lower as compared to water. Moreover, the highest uptake of the solution was nearly constant for immersion cycles 3 and 4 .

3.2. Effect of Cyclic Immersion on Tensile Strength. Tensile strength of the composite influenced by combined effect of material composition, liquid of immersion, cycle of immersion, and conditioning is shown in Figure 2. It is seen that the fibre loading clearly influenced tensile properties of the composites; increase in fibre loading caused reduction in tensile strength at all immersion cycle. The liquid absorbed caused fibre to become more ductile during pull-out due to Poisson's effect; that is, fibre length increased with decrease in diameter. 


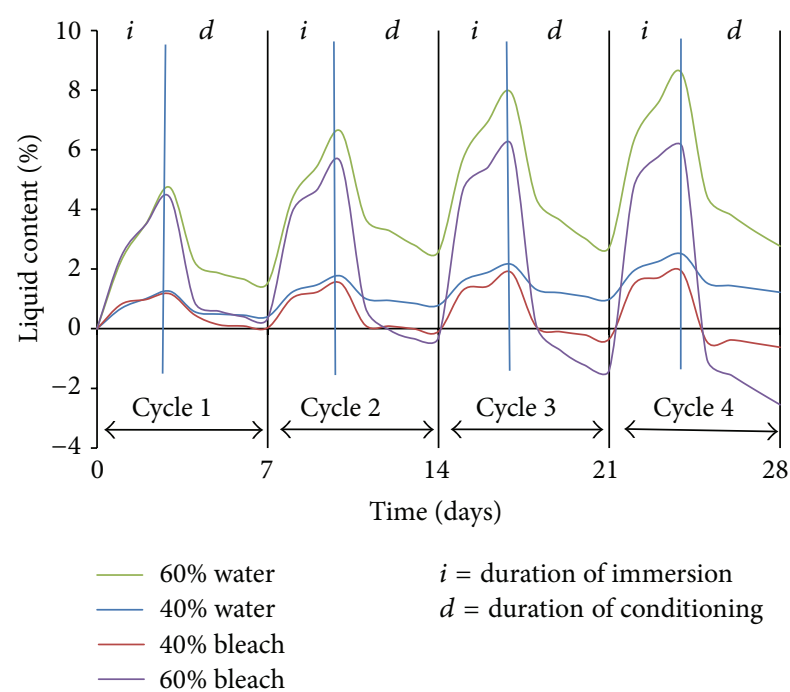

FIgure 1: Pattern of liquid content during immersion and conditioning of kenaf/PP composites.

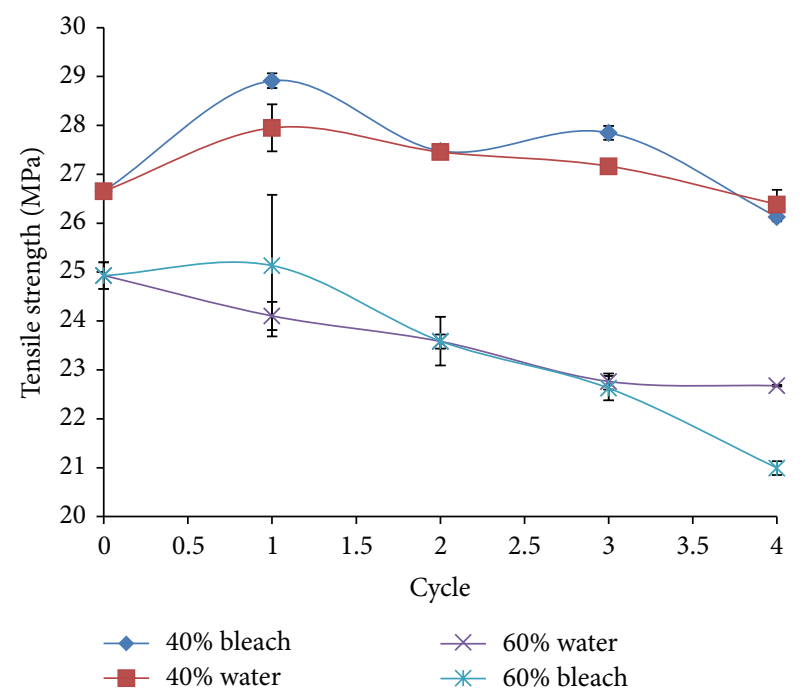

FIGURE 2: Tensile strength of PP composites with $40 \%$ and $60 \%$ of kenaf fibres loadings due to repeated immersion and drying.

The liquid also filled the gap between fibre and matrix and acted as lubricant during fibre pull-out. Residual stress might be produced due to uneven cooling or compaction during injection moulding. The early penetration of liquid, during dimensional expansion of fibre, might cause the fibre to release the residual stress exerted on it. However, the liquid could act as a lubricant when the amount of penetrated liquid increased.

3.3. Statistical Analysis on Tensile Strength. Statistical analysis is presented in Table 1. F-Test on three factors has been carried out to confirm the significance difference on tensile strength caused by compositions (composites with $40 \%$ and $60 \%$ fibre loadings), types of immersion liquid immersed into (water and bleach), and cycles (cycle 0 , cycle 1 , cycle 2 , cycle 3 , and cycle 4 ). The differences between parameters of composition and cycle were also significant. Thus, it was confirmed that fibre loading in the composites affected tensile strength of composites.

Although the $F$-Test indicated that the difference between cycles was significant, the actual difference between cycles was shown by Fisher's least significant difference (LSD) method as presented in Table 2. The significant increase in tensile strength during the first cycle of immersion was verified by statistical analysis (Table 2 ). Then, tensile strength dropped to a value similar to cycle 0 and cycle 3 . Possibly the residual stress, originating from processing of the composites, was released at early stage of immersion and thus improved tensile strength of the composite. However, as the immersions repeated, the liquid seeped between matrix and fibre and reduced the efficiency of loads transferred from matrix to the fibre that resulted in lower tensile strength of the composites.

In the analysis of liquid, $F$-Test in Table 3 shows that liquids of immersion (bleach and water) did not give significant difference regarding tensile strength of the composite, but there was significant interaction in $F$-Test between liquid and cycle (noted by Liquid $*$ Cycle in Table 1 ). Therefore, each cycle underwent $F$-Test to isolate the interaction effect. $F$ Tests results showed that liquid of immersion significantly affected tensile strength of composites only on cycle 4 . LSD analysis as shown in Table 3 reconfirmed significant difference between water and bleach in cycle 4 . Therefore, the degradation in cycles 2 to 3 was observed to be higher due to the effect of water whereas only on the fourth cycle the bleach affected tensile properties of kenaf/PP composites.

3.4. Effect of Repeated Immersion on Tensile Modulus. Modulus (stiffness) is another basic property of composites; the primary intention of filler incorporation is usually to increase the stiffness of the resultant material. However, moisture absorption could significantly reduce the modulus of the composites. Clear differences amongst the effects of fibre loading, immersion cycles, and liquid of immersion on tensile modulus of the composites are observed (Figure 3). As the immersion cycle was increased, a reduction in modulus with both liquid immersions was observed. However, this reduction in tensile modulus was drastic and significantly higher in composites with $60 \%$ fibre loading as compared to $40 \%$ fibre loading. The moisture uptake, due to the immersion process, changes the structure and properties of fibers and matrix and the interface between them [18]. Moreover, as fiber content was increased, water absorption was expected to be increased $[25,26]$. High fiber content in the composite leads to more water penetration into the interface through the micro cracks induced by swelling of fibers creating swelling stresses that led to composites failure [18]. The extent of strength and stiffness loss depends upon aging time and temperature also. Thwe and Liao [27] reported on bamboofiber composites that both the tensile strength and modulus decreased after aging in water at 25 and $75^{\circ} \mathrm{C}$ for prolonged period.

The composites immersed in bleach solution had lower modulus compared to water immersed composite samples. 
TABLE 1: ANOVA for tensile strength of kenaf/PP composite with $40 \%$ and $60 \%$ fibre loadings immersed in water and bleach.

\begin{tabular}{|c|c|c|c|c|c|}
\hline Source & DF & Sum of squares & Mean square & $F$ value & $\operatorname{Pr}>F$ \\
\hline Composition & 1 & 242.32 & 242.328 & 384.28 & $<0.0001$ \\
\hline Liquid & 1 & 0.7029 & 0.70291 & 1.11 & 0.2953 \\
\hline Composition $*$ Liquid & 1 & 0.0183 & 0.01831 & 0.03 & 0.8653 \\
\hline Cycle & 4 & 64.168 & 16.0421 & 25.44 & $<0.0001$ \\
\hline Composition $*$ Cycle & 4 & 17.019 & 4.25486 & 6.75 & 0.0001 \\
\hline Liquid $*$ Cycle & 4 & 11.245 & 2.81119 & 4.46 & 0.0032 \\
\hline Composition $*$ Liquid $*$ Cycle & 4 & 0.5062 & 0.12656 & 0.20 & 0.9370 \\
\hline Error & 60 & 37.836 & 0.63061 & & \\
\hline Corrected total & 79 & 373.82 & & & \\
\hline
\end{tabular}

TABLE 2: Means comparison among cycles for tensile strength.

\begin{tabular}{lclc}
\hline$t$ grouping & Mean & $N$ & Cycle \\
\hline A & 26.4623 & 16 & Cycle 0 \\
B & 25.7923 & 16 & Cycle 1 \\
B C & 25.4007 & 16 & Cycle 2 \\
C & 24.9134 & 16 & Cycle 3 \\
D & 23.7962 & 16 & Cycle 4 \\
\hline
\end{tabular}

TABLE 3: Compilation of mean comparison among liquid at each cycle.

\begin{tabular}{|c|c|c|c|}
\hline \multirow{2}{*}{ Cycle } & \multicolumn{3}{|c|}{ Liquid } \\
\hline & Water $(\mathrm{MPa})$ & Bleach (MPa) & Significant $(\mathrm{Y} / \mathrm{N})$ \\
\hline 0 & 25.7923 & 25.7923 & $\mathrm{~N}$ \\
\hline 1 & 26.026 & 26.8985 & $\mathrm{~N}$ \\
\hline 2 & 25.5191 & 25.2822 & $\mathrm{~N}$ \\
\hline 3 & 24.9653 & 24.8616 & $\mathrm{~N}$ \\
\hline 4 & 24.5308 & 23.0616 & $\mathrm{Y}$ \\
\hline
\end{tabular}

This is perhaps due to degradation of the natural fibers by bleach solution, which might have caused the fibermatrix interfacial debonding. The potential for fiber/matrix debonding and microcracking at the interface during wet/dry cycling of composites is dependent upon (among other factors) the dimensional stability of the fiber reinforcement in response to moisture fluctuations. Mohr et al. [28] showed that composites produced with fibers, which were wetted and dried prior to the mixing, exhibited superior dimensional stability compared to composites produced with fibers, which had never been dried. The bleached (i.e., low-lignin) composites exhibited accelerated progression of fiber mineralization as compared to unbleached fibers. Unbleached composites exhibited greater toughness, particularly for low numbers of wet/dry cycles. Without exposure, unbleached fiber composites exhibit greater flexural properties than bleached fiber composites.

3.5. Statistical Analysis on Tensile Modulus. Statistical analysis with $F$-Test in Table 4 showed that the difference between parameters of composition, liquid, and cycle was significant.

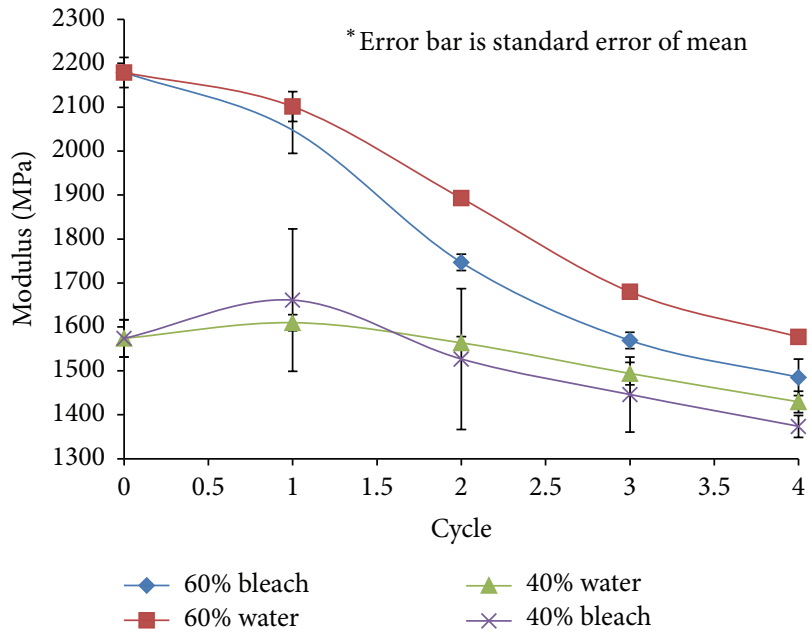

FIGURE 3: Tensile modulus of kenaf/PP composites with $40 \%$ and $60 \%$ fibre loadings due to repeated immersion and drying.

Since all the factors are significant, the interaction between the factors does not influence the results of ANOVA F-Test.

Both composition and liquid of immersion had only two parameters; the significant difference in Table 4 referred to composites with $40 \%$ and $60 \%$ fibre loadings and also water and bleach, respectively. On the other hand, cycle had five parameters and LSD test was used to compare which parameters were different. Table 5 showed that cycles 2, 3, and 4 differ from all cycles but cycles 0 and 1 do not differ from each other. This proved that significant degradation only started on cycle 2 onward.

The experiment confirmed that cycle of immersion, composition, and liquid of immersion had effects on tensile modulus of kenaf/PP composites. However, the cycle of immersion only affected tensile modulus of the composites on the second cycle onward.

3.6. Difference in Tensile Strength between Cyclic and Continuous Immersion. Result of tensile strength of composites with 40 and $60 \%$ fibre loading immersed in water and bleach is presented in Figure 4. Both types of immersion had caused degradation in composites with $60 \%$ fibre loading immersed in bleach. Tensile strength of composite with $40 \%$ 
TABLE 4: ANOVA for tensile modulus of 40 and $60 \%$ fibre loading kenaf/PP composites immersed in water and bleach.

\begin{tabular}{|c|c|c|c|c|c|}
\hline Source & DF & Sum of squares & Mean square & $F$ value & $\operatorname{Pr}>F$ \\
\hline Composition & 1 & 2057626 & 2057626 & 535.48 & $<0.0001$ \\
\hline Liquid & 1 & 48306 & 48306 & 12.57 & 0.0008 \\
\hline Composition $*$ Liquid & 1 & 19701 & 19701 & 5.13 & 0.0272 \\
\hline Cycle & 4 & 2117627 & 529407 & 137.77 & $<0.0001$ \\
\hline Composition $*$ Cycle & 4 & 644814 & 161203 & 41.95 & $<0.0001$ \\
\hline Liquid $*$ Cycle & 4 & 32139 & 8035 & 2.09 & 0.0931 \\
\hline Composition $*$ Liquid $*$ Cycle & 4 & 8649 & 2162 & 0.56 & 0.6906 \\
\hline Error & 60 & 230554 & 3843 & & \\
\hline Corrected total & 79 & 5159415 & & & \\
\hline
\end{tabular}

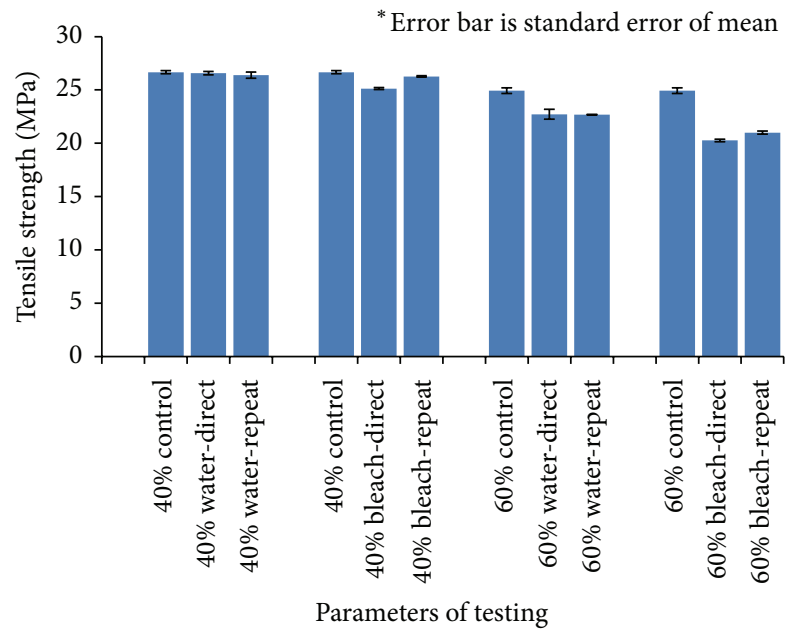

FIGURE 4: Difference of tensile strength between control, continuous (direct), and cyclic (repeat) immersion for 40 and 60\% fibre loading of kenaf/PP composites.

TABLE 5: Means comparison between cycles for tensile modulus.

\begin{tabular}{lccc}
\hline$t$ grouping & Mean & $N$ & Cycle \\
\hline A & 1876.53 & 16 & Cycle 0 \\
A & 1854.93 & 16 & Cycle 1 \\
B & 1682.55 & 16 & Cycle 2 \\
C & 1547.28 & 16 & Cycle 3 \\
D & 1466.32 & 16 & Cycle 4 \\
\hline
\end{tabular}

fibre loading immersed in water under continuous and cyclic immersion did not significantly differ. Tensile strength of composite with $40 \%$ fibre loading immersed in bleach was degraded under continuous immersion but, under cyclic immersion, tensile strength remains almost the same as composite without any immersion. It might be possible that conditioning process in cyclic immersion reduced the activity of bleach on the composites.

3.7. Differences in Tensile Modulus between Cyclic and Continuous Immersion. Both continuous and cyclic immersions had reduced tensile modulus of composites (refer to Figure 5). Composites with $40 \%$ fibre loading had loss around

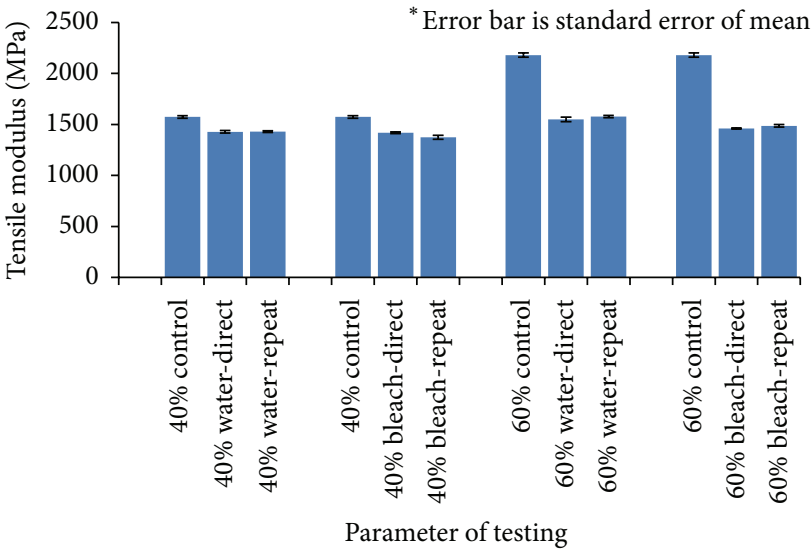

FIGURE 5: Difference of tensile modulus between control, continuous (direct), and cyclic (repeat) immersion for $40 \%$ and $60 \%$ fibre loading of kenaf/PP composite.

$100 \mathrm{MPa}$ of tensile modulus while composites with $60 \%$ fibre loading had loss around $600 \mathrm{MPa}$ of tensile modulus due to 12 days of immersion. Furthermore, composites under continuous and cyclic immersion had similar value of tensile modulus. Therefore, the reduction of tensile modulus is not influenced by type of immersions.

\section{Conclusions}

Both continuous and cyclic immersions in bleach affected the tensile strength of the kenaf fibre reinforced polypropylene composites. Increased number of cycles of immersion and conditioning caused degradation of tensile strength and modulus. However, continuous immersion in the bleach reduced the tensile strength of composites more compared to cyclic immersion.

\section{Conflict of Interests}

The authors declare that there is no conflict of interests regarding the publication of this paper. 


\section{Acknowledgment}

The authors would like to thank the Faculty of Engineering, Universiti Putra Malaysia, for all the supports to carry out this research.

\section{References}

[1] M. E. Hoque, M. A. I. M. Aminudin, M. Jawaid, M. S. Islam, N. Saba, and M. T. Paridah, "Physical, mechanical, and biodegradable properties of meranti wood polymer composites," Materials \& Design, vol. 64, pp. 743-749, 2014.

[2] K. Y. Tshai, A. B. Chai, I. Kong, M. E. Hoque, and K. H. Tshai, "Hybrid fibre polylactide acid composite with empty fruit bunch: chopped glass strands," Journal of Composites, vol. 2014, Article ID 987956, 7 pages, 2014.

[3] A. Stamboulis, C. A. Baillie, and T. Peijs, "Effects of environmental conditions on mechanical and physical properties of flax fibers," Composites, Part A: Applied Science and Manufacturing, vol. 32, no. 8, pp. 1105-1115, 2001.

[4] A. Stamboulis, C. A. Baillie, S. K. Garkhail, H. G. H. Van Melick, and T. Peijs, "Environmental durability of flax fibres and their composites based on polypropylene matrix," Applied Composite Materials, vol. 7, no. 5-6, pp. 273-294, 2000.

[5] M. Assarar, D. Scida, A. El Mahi, C. Poilâne, and R. Ayad, "Influence of water ageing on mechanical properties and damage events of two reinforced composite materials: flax-fibres and glass-fibres," Materials and Design, vol. 32, no. 2, pp. 788-795, 2011.

[6] R. V. Silva, E. M. F. Aquino, L. P. S. Rodrigues, and A. R. F. Barros, "Curaua/glass hybrid composite: the effect of water aging on the mechanical properties," Journal of Reinforced Plastics and Composites, vol. 28, no. 15, pp. 1857-1868, 2009.

[7] A. Athijayamani, M. Thiruchitrambalam, U. Natarajan, and B. Pazhanivel, "Effect of moisture absorption on the mechanical properties of randomly oriented natural fibers/polyester hybrid composite," Materials Science and Engineering A, vol. 517, no. 1-2, pp. 344-353, 2009.

[8] N. Sgriccia, M. C. Hawley, and M. Misra, "Characterization of natural fiber surfaces and natural fiber composites," Composites Part A: Applied Science and Manufacturing, vol. 39, no. 10, pp. 1632-1637, 2008.

[9] A. N. Fraga, E. Frullloni, O. DeLa Osa, J. M. Kenny, and A. Vázquez, "Relationship between water absorption and dielectric behavior of glass fiber reinforced unsaturated polyester resin," Journal of Composite Materials, vol. 41, no. 4, pp. 393402, 2007.

[10] S. T. Grant and L. W. Bradley, "In-situ observations in SEM of degradation of graphite/epoxy composite materials due to seawater immersion," Journal of Composite Materials, vol. 29, no. 7, pp. 852-867, 1995.

[11] K. Takemura, "Effect of water absorption on mechanical properties of hemp fiber reinforced composite," Key Engineering Materials, vol. 417-418, pp. 161-164, 2010.

[12] S. Panthapulakkal and M. Sain, "Studies on the water absorption properties of short hemp-glass fiber hybrid polypropylene composites," Journal of Composite Materials, vol. 41, no. 15, pp. 1871-1883, 2007.

[13] S. K. Najafi, H. Sharifnia, and M. Tajvidi, "Effects of water absorption on creep behavior of wood-plastic composites," Journal of Composite Materials, vol. 42, no. 10, pp. 993-1002, 2008.
[14] W. H. Haniffah, A. Khalina, and S. M. Sapuan, "Water retention in kenaf/polypropylene composites due to repeated immersion and drying conditions," Key Engineering Materials, vol. 471-472, pp. 438-443, 2011.

[15] H. N. Dhakal, Z. Y. Zhang, and M. O. W. Richardson, "Effect of water absorption on the mechanical properties of hemp fibre reinforced unsaturated polyester composites," Composites Science and Technology, vol. 67, no. 7-8, pp. 16747-1683, 2007.

[16] M. M. S. Nóbrega, W. S. Cavalcanti, L. H. Carvalho, and A. G. B. Lima, "Water absorption in unsaturated polyester composites reinforced with caroá fiber fabrics: modeling and simulation," Material Science and Engineering Technology, vol. 41, no. 5, pp. 300-305, 2010.

[17] M. A. Shenoy and D. J. D’Melo, "Effect of water on mechanical properties of unsaturated polyester-acetylated hydroxypropyl guar gum composites," Journal of Reinforced Plastics and Composites, vol. 28, no. 21, pp. 2561-2576, 2009.

[18] A. A. A. Rashdi, S. M. Sapuan, M. M. H. Ahmad, and M. Khalin, "Combined effects of water absorption due to water immersion, soil buried and natural weather on mechanical properties of kenaf fibre unsaturated polyester composites (KFUPC)," International Journal of Mechanical and Materials Engineering, vol. 5, no. 1, pp. 11-17, 2010.

[19] A. K. Bledzki, M. Letman-Sakiewicz, and M. Murr, "Influence of static and cyclic climate condition on bending properties of wood plastic composites (WPC)," Express Polymer Letters, vol. 4, no. 6, pp. 364-372, 2010.

[20] H. J. Kim and D. W. Seo, "Effect of water absorption fatigue on mechanical properties of sisal textile-reinforced composites," International Journal of Fatigue, vol. 28, no. 10, pp. 1307-1314, 2006.

[21] A. A. A. Rashdi, S. M. Sapuan, M. M. H. M. Ahmad, and A. Khalina, "Water absorption and tensile properties of soil buried kenaf fibre reinforced unsaturated polyester composites (KFRUPC)," Food Agriculture and Environment, vol. 17, no. 3-4, p. 909, 2009.

[22] P. Wambua, J. Ivens, and I. Verpoest, "Natural fibres: can they replace glass in fibre reinforced plastics?" Composites Science and Technology, vol. 63, no. 9, pp. 1259-1264, 2003.

[23] M. Tajvidi, S. K. Najafi, M. M. Shekaraby, and N. Motiee, "Effect of chemical reagents on the mechanical properties of natural fiber polypropylene composites," Polymer Composites, vol. 27, no. 5, pp. 563-569, 2006.

[24] C. Maier and T. Calafut, Polypropylene: The Definitive User's Guide and Databook, William Andrew, New York, NY, USA, 1998.

[25] P. W. France, W. J. Duncan, D. J. Smith, and K. J. Beales, "Strength and fatigue of multicomponent optical glass fibres," Journal of Materials Science, vol. 18, no. 3, pp. 785-792, 1983.

[26] S. L. LeVan and J. E. Winandy, "Effects of fire retardant treatments on wood strength: a review," Wood and Fiber Science, vol. 22, no. 1, pp. 113-131, 1990.

[27] M. M. Thwe and K. Liao, "Durability of bamboo-glass fibre reinforced polymer matrix hybrid composites," Composites Science and Technology, vol. 63, no. 3-4, pp. 375-387, 2003.

[28] B. J. Mohr, H. Nanko, and K. E. Kurtis, "Durability of kraft pulp fiber-cement composites to wet/dry cycling," Cement and Concrete Composites, vol. 22, no. 4, p. 4352, 2005. 

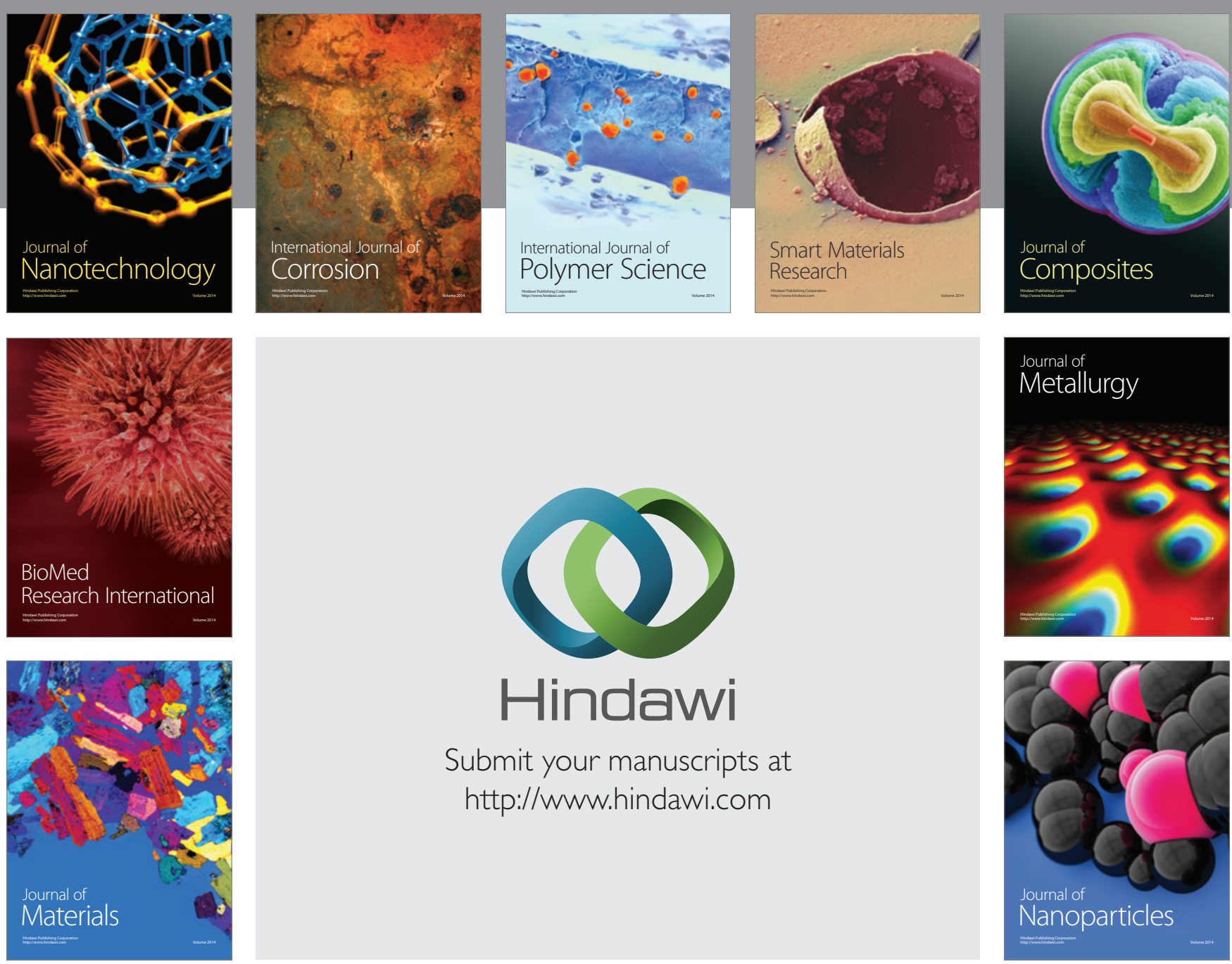

Submit your manuscripts at http://www.hindawi.com
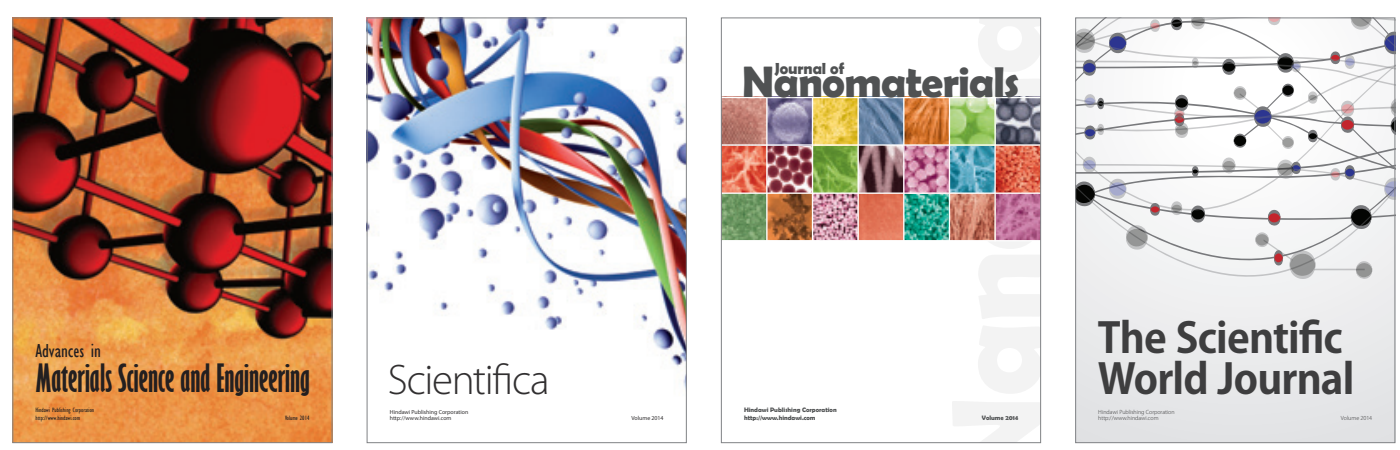

\section{The Scientific World Journal}
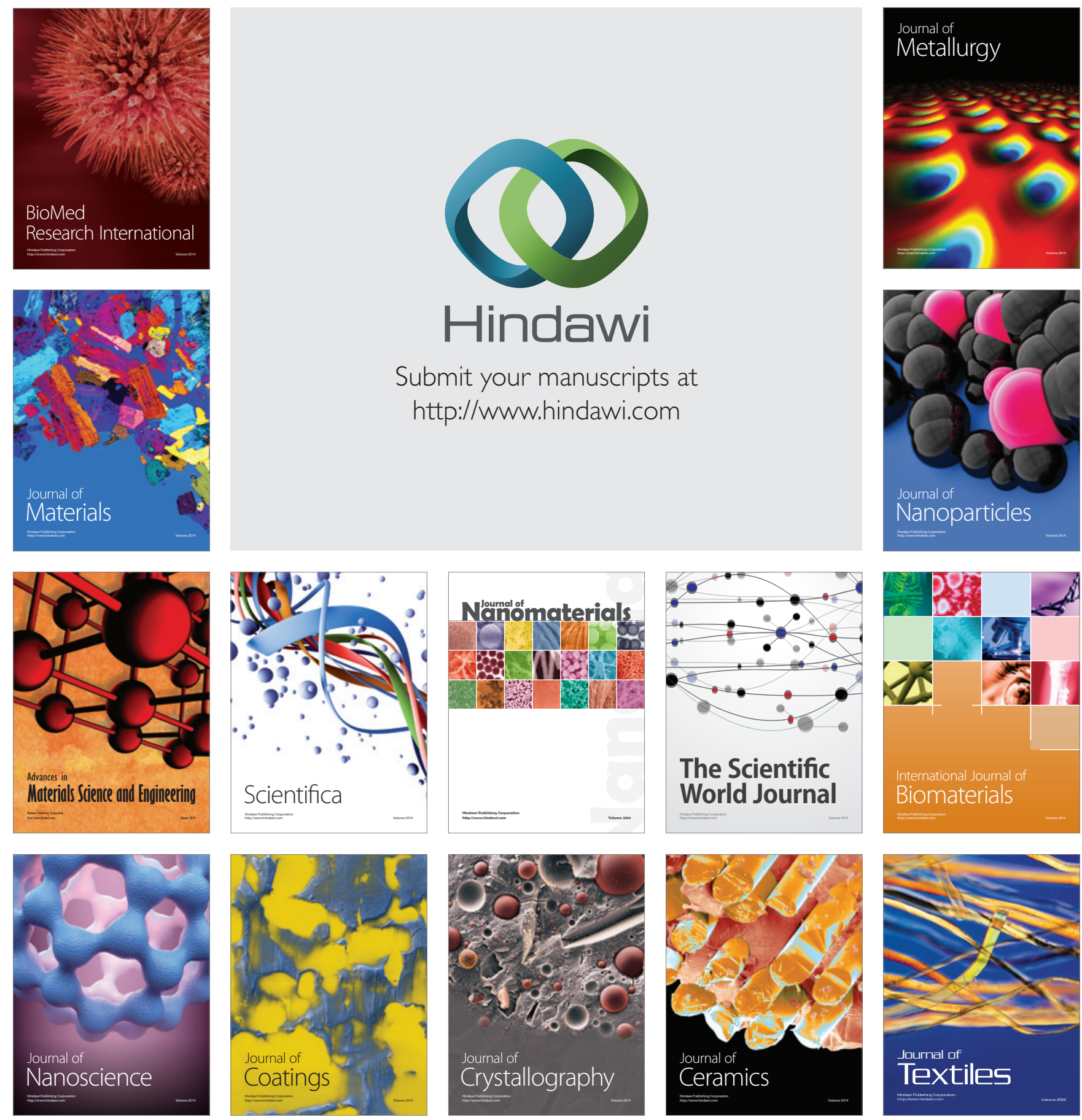